\title{
Prevalence of hepatic dysfunction in children with dengue fever
}

\author{
Siddappa F.D. ${ }^{1}$, Varsha Lakshman ${ }^{2}$, Madhu P.K. ${ }^{3}$ \\ ${ }^{1}$ Dr. Siddappa F.D., ${ }^{2}$ Dr. Varsha Lakshman, ${ }^{3}$ Dr. Madhu P.K, all authors are affiliated with Department of Paediatrics, \\ Karnataka Institute of Medical Sciences, Hubli, Karnataka, India.
}

Corresponding Author: Dr. Madhu P.K., Department of Paediatrics, Karnataka Institute of Medical Sciences, Hubli, Karnataka, India, E-mail: drmadhupk@gmail.com

\begin{abstract}
Background: Dengue is one disease entity with different clinical presentations and often with unpredictable clinical evolution and outcome. The degree of liver dysfunction in children with dengue infection varies from mild injury with elevation of transaminases to severe injury with jaundice and liver cell failure. This study was undertaken to find the prevalence of hepatic dysfunction in children admitted with Dengue fever. Methods: All children with serologically (dengue NS1 antigen detection and/or dengue IgM Mac ELISA) confirmed dengue fever aged between 2 months and 12 years were included in the study. Children with other etiologies of hepatitis were excluded. Liver function tests including bilirubin, transaminases and prothrombin time (PT) were done. Prevalence and severity of hepatic dysfunction were statistically analysed and compared between different categories of dengue fever severity. Results: Hepatic dysfunction was present in $35(67.30 \%)$ of the 52 children included in the study.About $85.71 \%$ of the children with dengue fever in the age group of 2 months to 1 year had hepatic dysfunction. In children with severe dengue, $83.3 \%$ had hepatic dysfunction.Raised liver enzymes in 32(61.53\%), hepatomegaly in 21(40.48\%), INR $\geq 1.5$ in $8(15.38 \%)$ and jaundice in $1(1.92 \%)$ children were the manifestations. None of the patients had acute hepatic failure or hepatic encephalopathy.Association of hepatomegaly with increasing disease severity was statistically significant $(p=0.0262)$. Hepatic dysfunction was present in $83.33 \%$ and $87.5 \%$ of children with abdominal pain and clinical fluid accumulation respectively. Of the 32 patients (61.53\%) with elevated transaminases, 18 (51.42\%) had elevated levels of AST alone, $40 \%$ had elevation of AST and ALT both. Conclusions: The prevalence of hepatic dysfunction among children with dengue fever is $67.30 \%$, more commonly observed in severe forms of dengue. Spectrum of hepatic involvement ranges from raised liver enzymes without jaundice to clinically evident jaundice. Hepatic dysfunction is commonly observed in children with abdominal pain and clinical fluid accumulation.
\end{abstract}

Keywords: Children, Dengue fever, Liver functions, Jaundice, Hepatitis, Transaminases

\section{Introduction}

Dengue is a major public-health concern throughout tropical and sub-tropical regions of the world. It is the most rapidly spreading mosquito-borne viral disease, with a 30 -fold increase in global incidence over the past 50 years. Although the full global burden of the disease is still uncertain, the patterns are alarming for both human health and the economy.

While dengue is a global concern, with a steady increase in the number of countries reporting the disease, currently close to $75 \%$ of the global population exposed to dengue are in the Asia-Pacific region[1]. An estimated 50 million dengue infections occur annually and approximately 2.5 billion people live in dengue

Manuscript received: $2^{\text {nd }}$ January 2019

Reviewed: $9^{\text {th }}$ January 2019

Author Corrected: $14^{\text {th }}$ January 2019

Accepted for Publication: $17^{\text {th }}$ January 2019 endemic countries [2]. Dengue constitutes a major cause of paediatric morbidity and mortality in South East Asian countries. In children, risk of death during secondary attack is nearly 15 folds higher than that of adults [3].

In humans, dengue infection causes a spectrum of illness ranging from relatively mild, non-specific viral syndrome to severe haemorrhagic disease and death. Expert consensus groups in Latin America, South-East Asia, and at WHO headquarters in Geneva, Switzerland in 2008 agreed that: "dengue is one disease entity with different clinical presentations and often with unpredictable clinical evolution and outcome"[2]. Unusual manifestations of dengue involving liver, heart and central nervous system have been reported. The degree of liver dysfunction in children with dengue 
infection varies from mild injury with elevation of transaminases to severe injury with jaundice and liver cell failure. Mechanisms of liver injury in dengue may be due to direct effects of the virus or host immune response on liver cells, circulatory compromise, metabolic acidosis and/or hypoxia caused by hypotension or localized vascular leakage inside the liver [4,5]. Pathological findings on liver biopsy includes hepatocellular necrosis, fatty change, kupffer cell hyperplasia and inflammatory cell infiltration $[6,7]$.

The incidence of hepatic dysfunction is more in dengue shock syndrome and Dengue hemorrhagic fever $[4,8]$. Rising of aminotransferase level occurs in the acute phase of the disease and elevated liver enzymes is an early marker of dengue infection [5]. Aminotransferase levels are also useful in predicting the occurrence of hepatic dysfunction and spontaneous bleeding [4]. In recent studies dengue infection was the most important cause of acute hepatic failure in children contributing $18.5 \%$ to $34.3 \%$ of the cases [4,9]. In developing country like India with huge population and poor health care facilities, it is important to know the prevalence of liver injury in children with dengue, so as to suspect and manage complications associated with liver injury despite lack of appropriate laboratory investigations.

This study was undertaken to know the prevalence, severity and spectrum of hepatic dysfunction in serologically confirmed cases of dengue fever in children admitted to a tertiary care hospital in north Karnataka.

\section{Methods}

Place of study: Department of Paediatrics, Karnataka Institute of Medical Sciences, Hubli, Karnataka

Type of study: Prospectiveobservational studyconducted from December 2016 to November 2017.

Sampling methods: All the clinically suspected cases of dengue fever aged between 2 months and 12 years as per the World Health Organization (WHO) guidelines, admitted during the study period were screened for dengue infection by Dengue NS1 antigen detection and/or positive dengue IgM Mac ELISA [2].

Inclusion criteria: All serologically confirmed cases of dengue fever (by dengue NS1 antigen detection and/or positive dengue IgM Mac ELISA) were included in the study.

Appendix-1: Age Specific range of biochemical indices and liver span.

\begin{tabular}{|c|c|c|}
\hline Parameters & Age range & Normal range \\
\hline Total bilirubin & 1month-adults & $<1.2 \mathrm{mg} / \mathrm{dl}$ \\
\hline Direct bilirubin & 1month-adults & $<0.2 \mathrm{mg} / \mathrm{dl}$ \\
\hline \multirow[t]{3}{*}{ Total protein } & 15 days-1yr & $5.1-7.3 \mathrm{~g} / \mathrm{dl}$ \\
\hline & $1-2 y r$ & $5.6-7.5 \mathrm{~g} / \mathrm{dl}$ \\
\hline & $3-16 y r$ & $6.0-8.0 \mathrm{~g} / \mathrm{dl}$ \\
\hline \multirow[t]{3}{*}{ Serum albumin } & 15 days- $1 \mathrm{yr}$ & $2.2-4.8 \mathrm{~g} / \mathrm{dl}$ \\
\hline & $1-2 \mathrm{yr}$ & $3.6-5.2 \mathrm{~g} / \mathrm{dl}$ \\
\hline & $3-16 y r$ & $3.6-5.2 \mathrm{~g} / \mathrm{dl}$ \\
\hline \multirow[t]{4}{*}{ Alanine aminotransferase } & $1-3 y r$ & $13-45 \mathrm{U} / \mathrm{L}$ \\
\hline & $4-6 y r$ & $10-25 \mathrm{U} / \mathrm{L}$ \\
\hline & $7-9 \mathrm{yr}$ & $10-35 \mathrm{U} / \mathrm{L}$ \\
\hline & $10-11 \mathrm{yr}$ & $10-35 \mathrm{U} / \mathrm{L}$ \\
\hline \multirow[t]{4}{*}{ Aspartate aminotransferase } & 0-10days & $47-150 \mathrm{U} / \mathrm{L}$ \\
\hline & 10days-24months & $9-80 \mathrm{U} / \mathrm{L}$ \\
\hline & $>24$ months female & $13-35 \mathrm{U} / \mathrm{L}$ \\
\hline & $>24$ months male & $15-40 / \mathrm{L}$ \\
\hline \multirow[t]{4}{*}{ Normal liver span } & Less than 1 year & $4-5 \mathrm{~cm}$ \\
\hline & $1-5$ year & $5-7 \mathrm{~cm}$ \\
\hline & $5-12$ years & $7-9 \mathrm{~cm}$ \\
\hline & More than 12 years & $9-12 \mathrm{~cm}$ \\
\hline
\end{tabular}


Exclusion criteria: Drug induced hepatitis and infective etiologies of hepatitis like malaria, enteric fever, hepatitis A and hepatitis B were excluded by history, examination and/or investigations.

Sample Collection: Informed consent was obtained from parents or guardians of the child included in the study. Detailed history was obtained and clinical examination was performed. Findings were entered in a predesigned proforma. Venous blood ( $5 \mathrm{ml}$ each) of all the children included in the study was collected in plain bottles, centrifuged and serum was separated.

Total serum bilirubin (TSB), direct serum bilirubin, indirect serum bilirubin, alanine transaminase (ALT), aspartate transaminase (AST), alkaline phosphatase (ALP), total proteins, serum albumin were measured using Trans Asian Biochemicals ERBA XL-300 fully computerised auto-analyser by advanced calorimetric methods. Prothrombin time (PT)/ international normalized ratio (INR) was measured by Trans Asian ERBA Coagulometer using Liquiplastin as a reagent on $2 \mathrm{ml}$ of blood collected in citrate tube to prevent the clotting process. Normal age specific ranges of biochemical indices of liver functionsand liver spanwere used to identify derangements [10,11]. (Appendix 1)

Scoring systems and definitions: Severity of dengue fever was classified as 1) dengue without warning signs, 2) dengue with warning signs and 3) severe dengue as per WHO [2].

The following definitions were used for each of the warning signs: Abdominal pain: abdominal tenderness and continuous pain, on some occasions diffuse. Persistent vomiting: more than three episodes of vomiting in 12 hours, preventing adequate oral hydration. Clinical accumulation of liquids: pleural effusion and ascites diagnosed clinically, confirmed with imaging techniques.

Mucosal bleeding: bleeding gums or conjunctiva, epistaxis, vaginal bleeding, bleeding from digestive, respiratory or urinary system (kidneys); mucosa defined as respiratory, vaginal, digestive, conjunctival and urinary tract mucosa. Lethargy: evaluated as an alteration of consciousness with a Glasgow score less than 15. Irritability: irritability or restlessness [12].

Liver dysfunction was considered to be present if any one of the findings like 1) elevated AST and/or ALT levels more than twice the upper limit of normal (ULN) for age with or without hepatomegaly, 2) jaundice or 3) INR $\geq 1.5$ was present.

Based on elevation of liver enzymes (AST and/or ALT), liver dysfunction was graded as 1) Liver function impairment was defined as an increase in AST and/or ALT level of at least twice the upper limit of normal, 2) Hepatitis was defined as an AST and/or ALT level at least four times higher than the upper limit of normal and 3) Severe hepatitis was defined as an AST and/or ALT level of at least 10 times higher than the upper limit of normal for age [6].

Statical Methods: Demographic, clinical and biochemical results were tabulated in Microsoft Excel sheet and statistical analysis was done using SPSS 20 software. Categorical data were presented as frequency percentage (\%) and continuous data were presented as mean and standard deviation (SD) or median and interquartile range. Categorical variables between the groups were compared using chi- square test and continuous variables were analysed using t-test for comparison of two means or one way ANOVA for multiple means. Appropriate statistical methods were employed for non-parametric data.

\section{Results}

A total of 103 children admitted with suspected dengue fever were screened for dengue NS1 antigen detection or positive dengue IgM by Mac ELISA. NS1 antigen and/or dengue Ig M was positive in 53 children, and serologically confirmed with dengue infection. One case was excluded due to coinfection with malaria. A total of 52 children were further analysed for presence of hepatic dysfunction. According to WHO classification of dengue fever severity, $26(50 \%)$ belonged to dengue without warning signs, $20(38.46 \%)$ belonged to dengue with warning signs and $6(11.54 \%$ ) belonged to severe dengue. Males were $34(65.38 \%)$ and females 18 (34.61\%) with a male to female ratio of 1.7:1. The mean age of occurrence of dengue fever was $6.36 \pm 3.9$ years. There were $7(13.46 \%)$ children in the age group of 2 months to 12 months, $16(30.77 \%)$ each in the age groups of $1-5$ years and 6- 10 years and $13(25 \%)$ children were more than 11 years old (Figure 1). 
Original Research Article

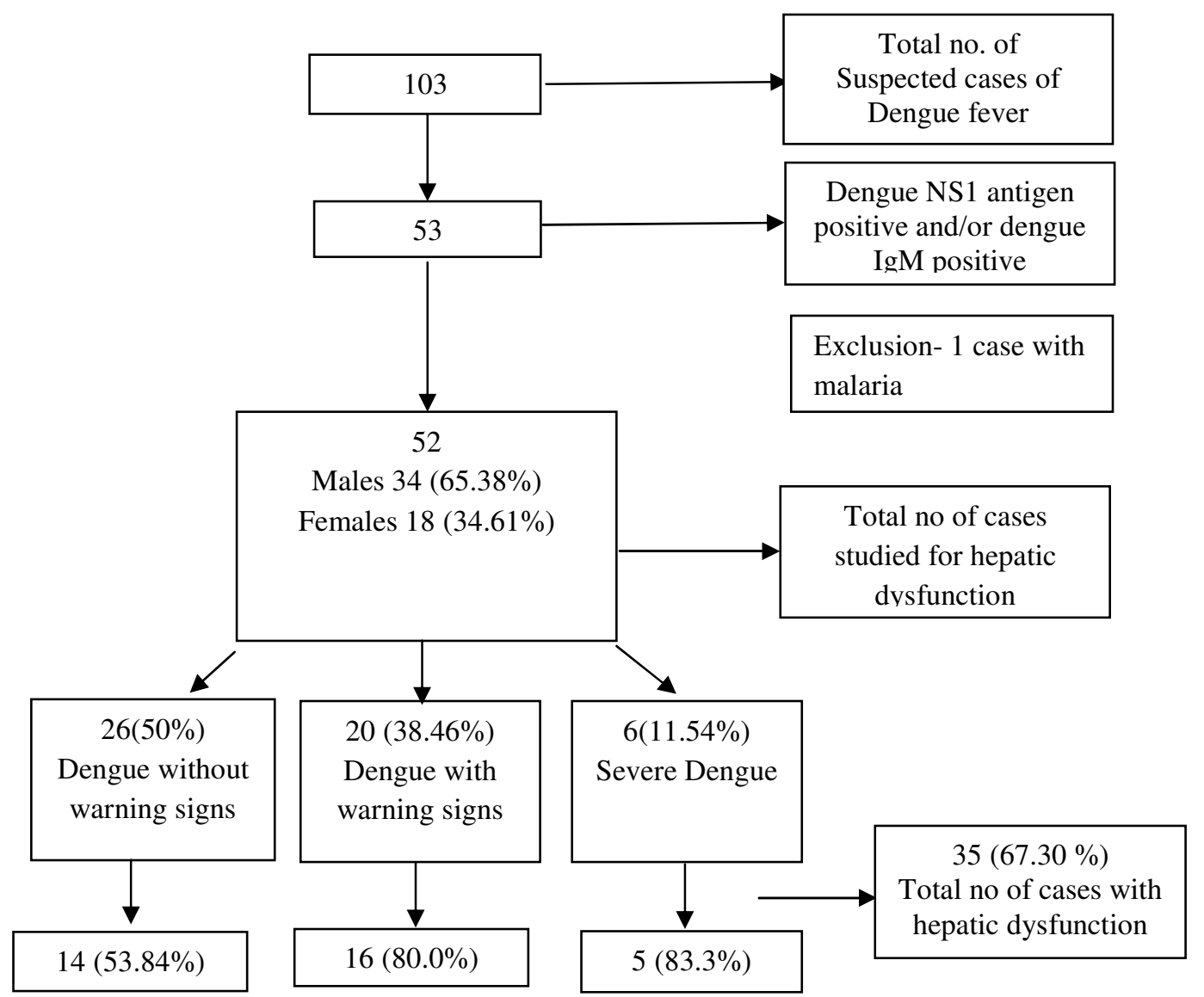

Figure-1: Dengue severity and proportion of children with hepatic dysfunction

Fever was the chief complaint presents in all the cases, followed by vomiting in 32(61.53\%), abdominal pain or tendernessin $18(34.61 \%)$ and myalgia in $12(23.07 \%)$. Clinical signs included flushing in $26(50 \%)$, hepatomegaly in $21(40.38 \%)$, rashes/petechiae in $11(21.15 \%)$, pedal edema in $3(5.77 \%)$ and icterus in $1(1.92 \%)$. Clinical fluid accumulation was noticed in $8(15.38 \%)$ and mucosal bleeding in $16(30.76 \%)$ children. Persistent vomiting defined as more than three episodes of vomiting in 12 hours, preventing adequate oral hydration was present in $14(26.92 \%)$ children (Table 1).

Table-1: Symptoms and signs of all Dengue cases $(n=52)$.

\begin{tabular}{|c|c|c|}
\hline Symptoms and signs & Total & Percentage (\%) \\
\hline Fever & 52 & 100 \\
\hline Vomiting & 32 & 61.53 \\
\hline Flushing & 26 & 50 \\
\hline Hepatomegaly & 21 & 40.38 \\
\hline Abdominal pain or tenderness & 18 & 34.61 \\
\hline Mucosal bleeding & 16 & 30.76 \\
\hline Persistent Vomiting & 14 & 26.92 \\
\hline Myalgia & 12 & 23.07 \\
\hline Rashes/ Petechiae & 11 & 21.15 \\
\hline Facial puffiness & 9 & 17.30 \\
\hline Clinical fluid accumulation & 8 & 15.38 \\
\hline Lethargy/Restlessness & 8 & 15.38 \\
\hline Shock & 4 & 7.69 \\
\hline Convulsions & 4 & 7.69 \\
\hline Pedal edema & 3 & 5.77 \\
\hline Icterus & 1 & 1.92 \\
\hline
\end{tabular}


Of the 52 cases, hepatic dysfunction was present in $35(67.30 \%)$ patients. Most common criteria met in the study to define hepatic dysfunction was elevated liver enzymes 32 (61.53\%), followed by hepatomegaly $21(40.48 \%)$, INR $\geq 1.5$ in $8(15.38 \%)$ children. Jaundice was present in $1(1.92 \%)$ child. None of the patients had acute hepatic failure or hepatic encephalopathy. Hepatomegaly was present in $21(40.38 \%)$ cases. All patients with severe dengue had hepatomegaly. Whereas $68.75 \%$ of dengue with warning signs and $35.71 \%$ of dengue without warning signs patients had hepatomegaly. This association of hepatomegaly with disease severity was statistically significant $(p=0.0262)$. (Table 2$)$

Table-2: Hepatic dysfunctions $(n=52)$.

\begin{tabular}{|c|c|c|}
\hline Hepatic dysfunctions & Number & Percentage (\%) \\
\hline Elevated liver enzymes $(>2$ times) & 32 & 40.53 \\
\hline Hepatomegaly & 21 & 15.38 \\
\hline INR $\geq 1.5$ & 1 & 1.92 \\
\hline Jaundice & 1 & \\
\hline
\end{tabular}

Hepatic dysfunction was observed in $64.70 \%$ of males and $72.22 \%$ of females. Among Children aged 2 months to 12 months, $85.71 \%$ had hepatic dysfunction. Prevalence of hepatic involvement was highest among children with severe dengue $(83.3 \%)$ followed by dengue with warning signs $(80 \%)$.

On analysis of sex, age and severity of dengue for development of hepatic dysfunction, the values were found to be not statistically significant. Hepatic dysfunction was present in $83.33 \%$ and $87.5 \%$ of children with abdominal pain and clinical fluid accumulation respectively. But this observation was not statistically significant. (Table 3 )

Table-3: Risk factor for hepatic dysfunction.

\begin{tabular}{|c|c|c|c|}
\hline Risk factors & $\begin{array}{l}\text { No. of children } \\
(n=52)\end{array}$ & $\begin{array}{l}\text { Hepatic dysfunction } \\
\text { present }(n=35)\end{array}$ & P value (chi square value) \\
\hline \multicolumn{4}{|c|}{ Sex } \\
\hline Male & 34 & $22(64.70 \%)$ & \multirow[t]{2}{*}{$0.5831(0.3022)$} \\
\hline Female & 18 & $13(72.22 \%)$ & \\
\hline \multicolumn{4}{|c|}{ Age } \\
\hline $2-12$ months & 7 & $6(85.71 \%)$ & \multirow{4}{*}{$0.4431(2.6812)$} \\
\hline $1-5 y r s$ & 16 & $10(62.5 \%)$ & \\
\hline 6-10yrs & 16 & $9(56.25 \%)$ & \\
\hline$>11 \mathrm{yrs}$ & 13 & $10(76.925)$ & \\
\hline \multicolumn{4}{|c|}{ Dengue severity } \\
\hline Dengue without warning signs & 26 & $14(53.84 \%)$ & \multirow{3}{*}{$0.5601(1.1592)$} \\
\hline Dengue with warning signs & 20 & $16(80.0 \%)$ & \\
\hline Severe dengue & 6 & $5(83.3 \%)$ & \\
\hline \multicolumn{4}{|c|}{ Warning signs } \\
\hline Abdominal pain or tenderness & 18 & $15(83.33 \%)$ & $0.1383(2.1957)$ \\
\hline Mucosal bleeding & 16 & $9(56.25 \%)$ & $0.4162(0.6609)$ \\
\hline Persistent Vomiting & 14 & $11(78.75 \%)$ & $0.4729(0.5152)$ \\
\hline Clinical fluid accumulation & 8 & $7(87.5 \%)$ & $0.3607(0.8352)$ \\
\hline Lethargy/Restlessness & 8 & $4(50 \%)$ & $0.4685(0.5254)$ \\
\hline
\end{tabular}


Original Research Article

Table-4: Association of AST and ALT enzyme levels with disease severity $(n=35)$

\begin{tabular}{|c|c|c|c|c|c|}
\hline & $\begin{array}{c}\text { Dengue without } \\
\text { warning signs } \\
(\mathbf{n = 1 4 )}\end{array}$ & $\begin{array}{c}\text { Dengue with } \\
\text { warning signs } \\
(\mathbf{n = 1 6})\end{array}$ & $\begin{array}{c}\text { Severe } \\
\text { dengue } \\
(\mathbf{n = 5})\end{array}$ & Total & p value \\
\hline \multicolumn{7}{|c|}{ AST } \\
\hline L2 fold rise & $2(14.28 \%)$ & $1(6.25 \%)$ & 0 & 3 & 17 \\
\hline
\end{tabular}

Table-5: Comparison of indicators of hepatic dysfunction with disease severity. $(n=35)$

\begin{tabular}{|c|c|c|c|c|}
\hline Indicator & $\begin{array}{c}\text { Dengue Without } \\
\text { Warning Signs } \\
(\mathbf{n = 1 4})\end{array}$ & $\begin{array}{c}\text { Dengue With } \\
\text { Warning Signs } \\
(\mathbf{n = 1 6})\end{array}$ & $\begin{array}{c}\text { Severe Dengue } \\
(\mathbf{n = 5})\end{array}$ & p value \\
\hline $\begin{array}{c}\text { Mean total bilirubin } \\
(\mathrm{mg} / \mathrm{dl})\end{array}$ & $0.65 \pm 0.34$ & $0.69 \pm 0.53$ & $0.38 \pm 0.11$ & 0.3435 \\
\hline Mean ALT(U/L) & $67.85 \pm 34.99$ & $120.38 \pm 112.01$ & $134 \pm 104.61$ & 0.1208 \\
\hline Mean AST(U/L) & $195.42 \pm 155.32$ & $211.56 \pm 164.28$ & $268.40 \pm 188.34$ & 0.6479 \\
\hline Mean PT (Seconds) & $14.99 \pm 1.44$ & $15.10 \pm 0.76$ & $17.06 \pm 1.35$ & $0.0056^{*}$ \\
\hline Mean INR & $1.24 \pm 0.22$ & $1.31 \pm 0.33$ & $1.62 \pm 0.41$ & 0.0623 \\
\hline
\end{tabular}

$* \mathrm{p}<0.05$

Out of 35 cases with hepatic dysfunction, 2 had normal values of AST and ALT. While, 18 (51.42\%) had elevated levels of AST, 40\% had elevation of AST and ALT both but none had elevation of ALT alone. All patients with severe dengue had AST rise $>2$ times the normal, whereas $92.9 \%$ and $87.5 \%$ of patients with dengue with warning signs and dengue without warning signs had AST levels $>2$ times the ULN respectively. $60 \%$ of cases with severe dengue, $50 \%$ of dengue with warning signs and $25 \%$ of dengue without warning signs had ALT levels $>2$ times the ULN. The mean levels of AST increased with the severity of the disease. Although patients with hepatomegaly had higher levels of liver enzymes (AST and ALT) than those without hepatomegaly, this was not statistically significant. Mean PT was more in severe dengue than in dengue with warning signs and dengue without warning signs with statistically significant association $(\mathrm{p}<0.05)$. INR $\geq 1.5$ was noted in 8 patients, which is more in severe dengue than other groups. (Table 4) (Table 5)

\section{Discussion}

Dengue fever (DF) is known to affect several systems in the human body. Liver is the commonest organ to be involved in dengue. Hepatic dysfunction is a wellrecognized feature of dengue infection and varying degree of dengue hepatitis is seen. The spectrum of involvement includes asymptomatic elevation of hepatic transaminases to occurrence of severe manifestation in form of acute liver cell failure (ALF)[5].

In our study 35 (67.30\%) children with dengue had one or the other hepatic manifestations (jaundice, hepatomegaly, raised serum tranaminases and/or INR $>1.5$ ).
Selvanet al observed hepatic dysfunction in 30.6\%[4]. Hepatic dysfunction in our study was observed in $64.70 \%$ of males and $72.22 \%$ of female children with dengue. This observation was not statistically significant. In children aged 2 months to 12 months with dengue, $85.71 \%$ had hepatic dysfunction.

Though not statically significant, this observation could be due to increased vulnerability of liver cells to various insults during infancy. Prevalence of hepatic dysfunction increases with severity of dengue infection. As observed in our study, $80 \%$ and $83.3 \%$ of children 
who had dengue with warning signs and severe dengue had hepatic dysfunction respectively. Jaundice in our study was reported in only one case, while in other studies incidence of jaundice ranges from 0-27.5\% $[4,13,14]$. Hepatomegaly is an important clinical sign observed in our study seen. Total of 21(40.38\%) children with hepatic dysfunction had hepatomegaly.

All the patients $(100 \%)$ with severe dengue had hepatomegaly, which is statistically significant ( $p=0.0262$ ) when compared to less severe forms of dengue infection. Jagadish kumaret al reported $21 \%$ of cases of dengue fever (DF) and $48 \%$ of dengue hemorrhagic fever (DHF) cases had hepatomegaly [13].

Wahid et al observed a slightly higher incidence of hepatomegaly in DHF $(60 \%)$ group compared to DF $(40 \%)$ group in their study [7]. A study by Tambolkaret al observed hepatomegaly in $69 \%$ with dengue fever and $100 \%$ with severe Dengue [15]. These findings suggest that hepatomegaly may be used as a predictor for assessing the hepatic dysfunction in severe forms of dengue.

Ascites was seen in $8(15.38 \%)$ patients in our study. In a study by Selvanet al $35.6 \%$ had ascites while Singh et alreported ascites in $23 \%$ cases [4,16]. In our study none of the patients had hepatic encephalopathy or acute liver failure. While Selvanet alreported that $4.5 \%$ of their cases had hepatic encephalopathy [4].

Mean AST and ALT levels were more in severe dengue (268.40 $\pm 188.34 \mathrm{U} / \mathrm{L}$ and $134 \pm 104.61 \mathrm{U} / \mathrm{L})$ than DF with warning signsand DF without warning signs. But this observation was not statistically significant.26.92\% and $61.53 \%$ of patients had ALT and AST rise more than 2 folds respectively. In a study by Jagadish kumar et al, mean AST and ALT levels increased with severity of the disease and rise of enzymes were more in dengue shock syndrome (DSS) and dengue hemorrhagic fever (DHF) compared to DF [13]. A similar findings was noted by Tambolkar S A et al [15].

We found more than 10 fold rise in AST in 20\% of severe dengue, $12.5 \%$ of DF with warning signs and $14.28 \%$ of DF without warning signs. Only in $6.25 \%$ of DF with warning signs there was elevation of ALT more than 10 folds. Jagadish Kumaret alreported more than 10 fold rise of AST in $44 \%$ of DSS, $22.8 \%$ of DHF and $3.4 \%$ of DFcases [13]. Tenfold rise of ALT was noted in $16 \%$ of DSS, $7.7 \%$ of DHF and $0 \%$ of DF.

This indicates that severity of hepatitis dysfunction is proportional to the severity of dengue infection.
Elevation of AST was more compared to ALT in the present study and similar observations was made by others also $[4,8,13,16]$. AST rise more than ALT in dengue, probably due to multi-organ affection in dengue, hence other sources of AST contributes to its rise (erythrocytes, cardiac, skeletal, renal, brain). This differs from pattern seen in viral hepatitis, in which ALT levels are usually more than or equal to AST levels.

There was no significant difference in mean TSB levels between disease groups. Only one patient of dengue with warning signs had TSB of $2.4 \mathrm{mg} / \mathrm{dl}$. Singh et al observed mean TSB in severe dengue to be significantly more compared to DF with warning signs and without warning signs [16].

Bleeding manifestations are an important clinical feature in DF. Mostly it is due to the low platelet count seen in these patients. But a significant proportion of bleeding manifestations are due to the presence of coagulopathy associated with DF. In our study bleeding in the form of hemetemesis, epistaxis, malena were reported among $30.76 \%$ of cases. Mean PT was more in severe dengue group compared to DF with or without warning signs with statistical significance (pvalue0.0056).

INR $>1.5$ were found in $8(15.38 \%)$, more with severe dengue. In a study by Singhet al the PT/INR was deranged in $11.11 \%$ patients [16]. In severe dengue and dengue fever with warning sign groups, deranged PT/INR were $54.54 \%$ and $4.34 \%$ respectively.

Similar findings were noted by JagadishKumaret al [13]. Derangementof coagulation profile as a result of hepatic dysfunction increases with severity of disease contributing to increased bleeding manifestations observed in these patients.

\section{Conclusion}

The prevalence of hepatic dysfunction among children aged 2 months- 12 years with dengue fever is $67.30 \%$.

This is more among severe dengue than with less severe forms. Spectrum of hepatic involvement varies from anicteric raised liver enzymes to clinically evident jaundice.

Hepatomegaly was the most important clinical sign seen in $40.38 \%$. AST was more commonly elevated than ALT in severe forms of dengue. Hepatic dysfunction was more common in children with abdominal pain and 
clinical fluid accumulation.Derangement of coagulation profile increased with severity of the disease. Thus, in resource limited settings presence of warning signs like abdominal pain or clinical fluid accumulation and severe forms of dengue should arouse the suspicion of hepatic dysfunction as dengue hepatitis is primarily anicteric.

\section{What is already known?}

Liver is the commonest organ to be involved in dengue. Hepatic dysfunction is a well-recognized feature of dengue infection and varying degree of dengue hepatitis is seen.

\section{What this study add to existing knowledge?}

In resource limited settings presence of warning signs like abdominal pain or clinical fluid accumulation and severe forms of dengue should arouse the suspicion of hepatic dysfunction as dengue hepatitis is primarily anicteric.

\section{Contributions by authors}

(1) First author: The conception and design of the study, drafting the article and final approval of the version to be submitted.

(2) Second author: Acquisition of data, analysis and interpretation of data.

(3) Corresponding author: Statistical analysis, revising for important intellectual content and correspondence.

\section{Acknowledgements}

\section{Declarations}

Funding: Nil, Conflict of interest: None initiated, Perission from IRB: Yes

\section{References}

1. World Health Organization. Global strategy for dengue prevention and control 2012-2020. WHO Geneva : World Health Organization; 2012.

2. World Health Organization. Dengue: Guidelines for Diagnosis, Treatment, Prevention and Control: New Edition. Geneva: World Health Organization. 2009.

3. Alam, A., Sadat, S., Swapan, Z., Ahmed, A., Karim, M., Paul, H., \& Zaman, S. (1). Clinical Profile of Dengue Fever in Children. Bangladesh Journal of Child Health, 33(2), 55-58. doi:10.3329/bjch.v33i2.5678.
4. Selvan T, Saravanan P, Nagaraj MV, Tudu MN. Study of hepatic dysfunction of dengue fever in children. Int J ContempPediatr.2017;4(3): 901- 4. doi: 10. 18203/2349-3291.ijcp20171695.

5. Bandyopadhyay D, Chattaraj S, Hajra A, et al. A Study on Spectrum of Hepatobiliary Dysfunctions and Pattern of Liver Involvement in Dengue Infection. J Clin Diagn Res. 2016 May;10(5):OC21-6. doi: 10.7860/ JCDR/ 2016/16946.7784. Epub 2016 May 1.

6. Martínez Vega R, Phumratanaprapin W, Phonrat B, et al. Differences in Liver Impairment Between Adults and Children with Dengue Infection. Am J Trop Med Hyg. 2016 May 4;94(5):1073-9. doi: 10.4269/ajtmh.150507. Epub 2016 Mar 14.

7. Wahid SF, Sanusi S, Zawawi MM, et al. A comparison of the pattern of liver involvement in dengue hemorrhagic fever with classic dengue fever. Southeast Asian J Trop Med Public Health. 2000 Jun; 31 (2):259-63.

8. Wiwanitkit V. Liver dysfunction in Dengue infection: an analysis of the previously published Thai cases. J Ayub Med Coll Abbottabad. 2007 Jan-Mar;19(1):10-2.

9. Kumar R, Tripathi P, Tripathi S, et al. Prevalence of dengue infection in north Indian children with acute hepatic failure. Ann Hepatol. 2008 Jan-Mar;7(1):59-62.

10. Engorn B, FlerlageJ,Johns Hopkins Hospital. Children's Medical and Surgical Center. The Harriet Lane handbook: a manual for pediatric house officers. 20th ed. Philadelphia, PA: Mosby/Elsevier; 2014.

11. Parthasarathy A, Menon P. IAP Textbook of pediatrics. 4th ed. New Delhi: Jaypee Brothers, Medical Publishers Pvt. Limited; 2008.

12. Narvaez F, Gutierrez G, Pérez MA, et al. Evaluation of the traditional and revised WHO classifications of Dengue disease severity. PLoSNegl Trop Dis. 2011 Nov; 5 (11):e1397. doi: 10.1371/journal.pntd.0001397. Epub 2011 Nov 8.

13. Jagadishkumar $\mathrm{K}$, Jain $\mathrm{P}$, Manjunath $\mathrm{VG}$, et al. Hepatic involvement in dengue Fever in children. Iran $\mathbf{J}$ Pediatr. 2012 Jun;22(2):231-6.

14. Mohan B, Patwari AK, Anand VK. et al. Hepatic dysfunction in childhood dengue infection. J Trop Pediatr. 2000 Feb; 46(1):40-3. doi: 10.1093/tropej/ 46.1.40. 
15. Tambolkar SA, Mishra A, Joshi H, Agarkhedkar S. Liver Functions as a marker of severity of disease in children with Dengue fever. Webmed Central PAEDIATRICS 2015; 6(8):WMC004962
16. Singh S, Meena JK, Verma CR, Bhaskar V. A hospital-based study of hepatic dysfunction in children with dengue fever. Asian Pacific J Trop Dis 2015;5 (12): 964-7.

\section{How to cite this article?}

Siddappa F.D, Varsha Lakshman, Madhu P.K. Prevalence of hepatic dysfunction in children with dengue fever. Int J Pediatr Res. 2019;6(01):8-16.doi:10.17511/ijpr.2019.i01.02 